\title{
Intravascular application of electrocautery in a rabbit model of abdominal aortic endarterectomy
}

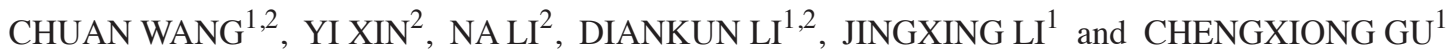 \\ ${ }^{1}$ Department of Cardiac Surgery, Beijing Anzhen Hospital, Capital Medical University; \\ ${ }^{2}$ Beijing Institute of Heart Lung and Blood Vessel Diseases, Beijing 100029, P.R. China
}

Received March 9, 2017; Accepted April 7, 2017

DOI: $10.3892 /$ etm.2017.4460

\begin{abstract}
Effective therapies for preventing perioperative complications such as thrombosis andinflammationaftercoronary endarterectomy (CE) are lacking. Electrocoagulation electrotomes have been routinely used in surgery for their cutting, clotting, and hemostatic properties. As strong flattening tools, their electrocautery function may prevent mechanical intimal-adventitial injury to arterial circulation and attenuate stenosis. The present study investigated the effects of intravascular application of electrocautery on ameliorating inflammation and thrombosis in a rabbit model of abdominal aortic endarterectomy. New Zealand rabbits were randomly divided into the sham, control (endarterectomy), and study (endarterectomy + electrocautery) groups with 10 in each group. Abdominal aortas were partially blocked and intima was removed. Electrocautery was performed with an electrocoagulation electrotome through the entire blocked vessel lumen. Vascular ultrasound parameters, molecular biological and histological characteristics of the abdominal aorta including vascular diameter, blood flow velocity, serum interleukin-6 (IL-6) and tumor necrosis factor- $\alpha$ (TNF- $\alpha$ ) levels, and apoptosis rate of vascular endothelial cells (ECs) were evaluated postoperatively by vascular Doppler ultrasound, ELISA, real-time RT-PCR, flow cytometry, and immunofluorescence at various time points. Compared with the endarterectomy + electrocautery group, the isolated endarterectomy group had significantly increased levels and gene expression of TNF- $\alpha$ and IL- 6 $(\mathrm{P}<0.05)$, and rates of apoptosis of vascular ECs $(\mathrm{P}<0.05)$, with gradual vascular stenosis and decreased blood flow velocity. In conclusion, intravascular application of electrocautery has favorable short-term effects on the abdominal
\end{abstract}

Correspondence to: Dr Chengxiong Gu, Department of Cardiac Surgery, Beijing Anzhen Hospital, Capital Medical University, 2 Anzhen Road, Chao Yang, Beijing 100029, P.R. China

E-mail: ag6y58@163.com

Key words: endarterectomy, electrocautery, thrombosis, inflammatory response aorta and can reduce inflammation in a rabbit model of abdominal aorta endarterectomy. Long-term anti-inflammatory and anti-thrombotic effects on arterial remodeling and the clinical value of electrocautery in CE remain to be determined.

\section{Introduction}

Over the past ten years, patients who underwent coronary artery bypass grafting (CABG) often presented with complications such as diffuse coronary diseases and multiple comorbidities (1). For patients with severe diffuse coronary disease, CABG combined with coronary endarterectomy (CE) has been demonstrated to be an effective approach for complete revascularization $(1,2)$. However, the short-term clinical outcomes from $\mathrm{CE}$ appears to be worse compared with $\mathrm{CABG}$ alone (1). Soylu et al, reported that CABG combined with CE markedly increased perioperative myocardial infarction (MI) and postoperative inflammation (2). In addition to inflammation and MI, 30-day mortality is a severe postoperative complication associated with CE (3). Furthermore, a 30-day mortality is also a severe postoperative complication associated with CE (4). Therefore, the development of the effect of CE and the addition of the subsequent optimal treatment has been significant in coronary surgery field. Electrocautery is a routine surgical option for various clinical requirements. Hence, the design of $\mathrm{CE}$ combined with electrocautery has been emerging to further distribute coronary disease treatment in this research. The aim of this study is to investigate the mechanism and optimize the therapeutic effect of electrocautery using a rabbit model of abdominal aortic endarterectomy.

\section{Materials and methods}

Ethics statement. A total of 30 healthy male white New Zealand rabbits, weighing between $2.5-3.6 \mathrm{~kg}$, were purchased from the Animal Center of Capital Medical University (Beijing, China). We conducted animal experiments according to the Animal Management Rules of the Ministry of Health of China and the Guidance for the Care and Use of Laboratory Animals (National Institutes of Health). All animals used for experiments were cared for according to these guidelines. The present study was approved by the Ethics Committee of the Animal Center of Beijing Anzhen Hospital. 


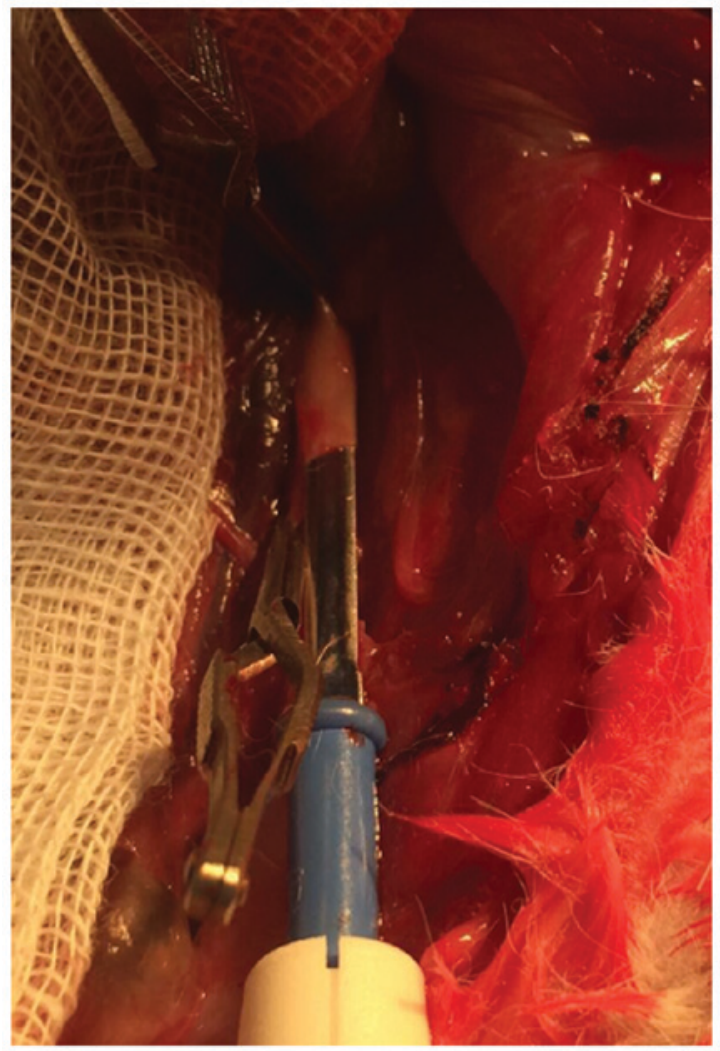

Figure 1. Procedure of electrocautery.

Preliminary experiments for establishment of electrocautery parameters. Five male rabbits housed at $25^{\circ} \mathrm{C}$ with $50 \%$ humidity and with free access to food and water were used for preliminary experiments. An operating microscope was used during surgery under aseptic conditions. After fasting for $6 \mathrm{~h}$, the rabbits were anaesthetized with $3 \%$ pentobarbital sodium via ear vein injection. Only those that presented with airway occlusion were placed on ventilation. Chest movement and respiratory rates were closely observed during surgery. The color of the oral mucosa, lip, and the conjunctival membranes was evaluated to reflect blood oxygenation. After airway occlusion developed, the airways of rabbits were opened and connected to a ventilator (DW: 3000B; Nanjing Jiancheng Co. Ltd., Nanjing, China). The surgical site over the abdominal aorta was anaesthetized by spreading $2 \%$ lidocaine $(5 \mathrm{ml})$ on the skin. Next, the abdominal aorta was exposed and heparin was injected via the ear vein at a dose of $700 \mathrm{IU} / \mathrm{kg}$. We placed Bulldog hemostatic clamps on the abdominal aorta to stop blood flow temporarily. The model of abdominal aortic endarterectomy was performed by denudation in the control and study groups as previously described $(5,6)$. To confirm the establishment of the model of electrocautery, the blocked abdominal aorta was partially incised and electrocautery via an electrocoagulation electrotome was performed through the entire lumen with the following parameters: $1 \mathrm{~W}+1 \mathrm{~S}$, $1 \mathrm{~W}+2 \mathrm{~S}, 2 \mathrm{~W}+1 \mathrm{~S}$ and $2 \mathrm{~W}+2 \mathrm{~S}$ (Fig. 1). The rest of the blocked aorta was cut off and split lengthways. Next, electrocautery was performed with each parameter for macroscopic observation (Fig. 1). The surgically treated vessels were harvested, and frozen sections were prepared for immunofluorescence staining to evaluate the results of electrocautery.
The rabbit model of abdominal aortic endarterectomy + electrocautery. Thirty rabbits were randomly divided into three groups as follows ( $n=10 /$ group): the sham and control groups (abdominal aortic endarterectomy by balloon catheter), and the study group (abdominal aortic endarterectomy by balloon catheter treated with electrocautery). The surgical procedures were performed as described above with optimal electrocautery parameters determined from the preliminary experiments. In the control and study groups, the incisions were anastomosed with mattressed 8-0 prolene sutures (Johnson \& Johnson, New Brunswick, NJ, USA). The surgical procedures for arterial blocking were completed within $30 \mathrm{~min}$ to avoid lower limb ischemia. After completing anastomoses, we released the hemostatic clamps at the abdominal aorta and then carefully examined the quality of anastomoses. The wounds were then closed. In the sham group, the abdominal incision was the operative site. In all groups, penicillin (4 million IU) and heparin $(700 \mathrm{IU} / \mathrm{kg}$ ) were administered daily for the first 3 days after surgery. Each rabbit received intragastric treatment with aspirin $(12.5 \mathrm{mg} /$ day $)$ until euthanasia.

Vascular histology. Rabbits were euthanized 3 weeks after surgery. To remove blood, animals were perfused with saline supplemented with heparin. The surgically treated aortas from the control and study groups were dissected and embedded in OCT. Vascular tissue sections $(7 \mu \mathrm{m})$ were prepared and stained with DAPI. Next, images were obtained and analyzed with a Nikon Nie fluorescence inversion microscope system (Nikon, Tokyo, Japan). The degree of endothelial injury and vascular morphology regarding pathological changes were determined.

Measurement of ultrasound parameters in the abdominal aorta. Blood flow velocity and arterial diameter were measured on postoperative 1, 3, 7, and 20 days using a flow QC meter (Vevo 2100; Fujifilm VisualSonics Inc., NY, USA). Using pentobarbital sodium (3\%), rabbits were anaesthetized and the abdominal aorta was exposed. The probe was placed on the abdominal aorta for measurement of vascular diameter and maximum blood flow velocity (Fig. 2).

Rate of apoptosis of vascular endothelial cells (ECs). Vascular tissues harvested at the various time points were digested with $2.5 \%$ trypsin for $10 \mathrm{~min}$, and the reaction was stopped by addition of FBS. The samples were centrifuged at $800 \mathrm{x} g$ for $5 \mathrm{~min}$. Next, tissues were incubated with $5 \mu \mathrm{l}$ of annexin $\mathrm{V}$ and propidium iodide (PI). Samples were then analyzed by flow cytometry.

ELISA for interleukin-6 (IL-6) and tumor necrosis factor- $\alpha(T N F-\alpha)$. Blood samples were collected from the ear vein and centrifuged at 3,000 rpm for $15 \mathrm{~min}$. ELISA was performed according to the manufacturer's instructions (R\&D, NY, USA). A total of $50 \mu \mathrm{l}$ of serum was used for the assay. Measurements were performed in triplicate. OD values at $450 \mathrm{~nm}$ were measured using a plate reader (Bio-Rad, NY, USA).

Quantitative polymerase chain reaction ( $q R T-P C R)$. We used a TRIzol kit from Life Technologies (Grand Island, NY, 


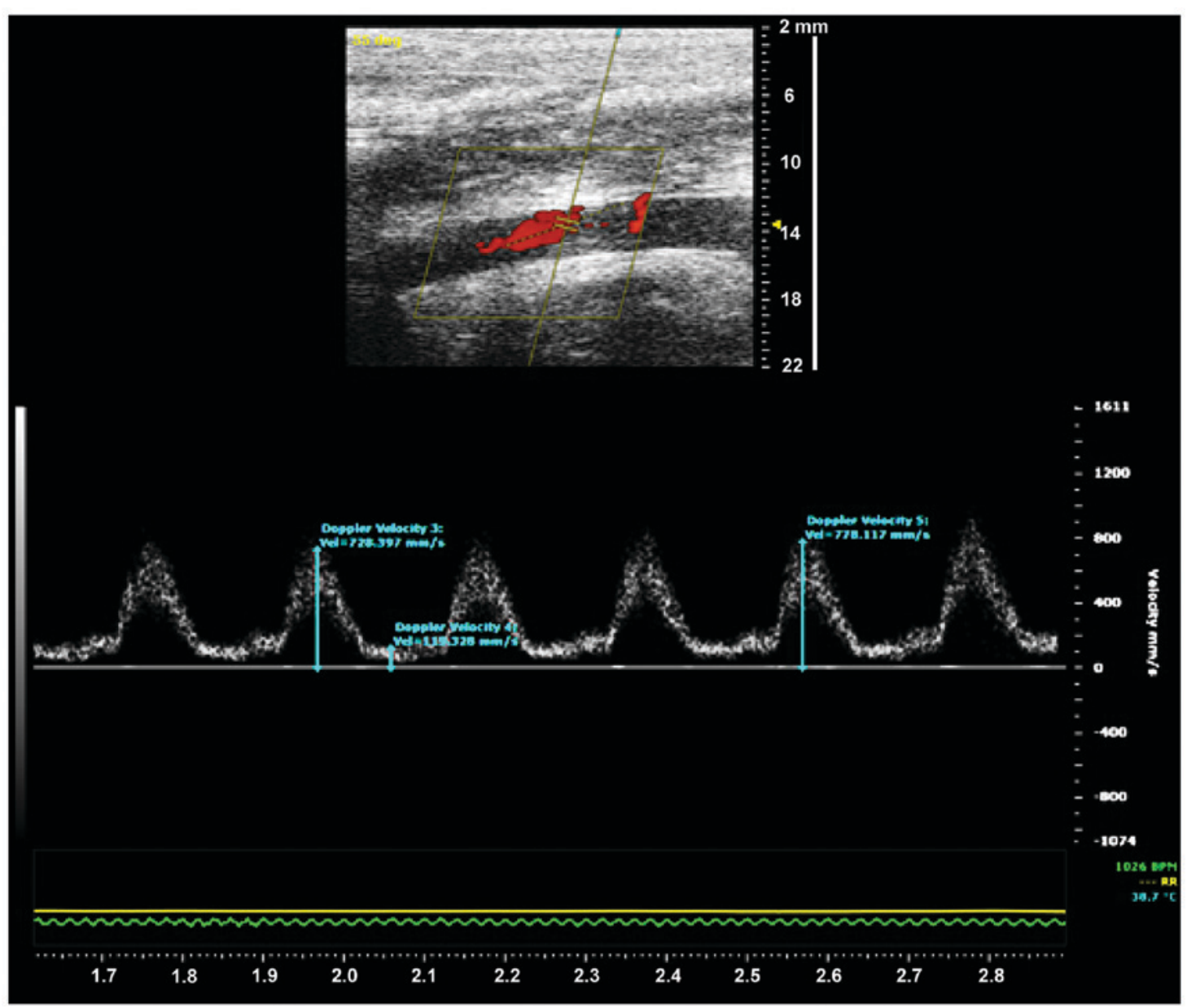

Figure 2. Measurement of blood flow velocity and artery diameter.

Table I. The sequence of PCR primers.

\begin{tabular}{llc}
\hline Gene & \multicolumn{1}{c}{ DNA sequence (5'-3') } & $\begin{array}{c}\text { Product } \\
\text { size (bp) }\end{array}$ \\
\hline TNF- $\alpha$ & U: ACCCTCACACTCAGATCATCTTCT & 422 \\
& D: CAGATTGACCTCAGCGCTGAGTTG & \\
IL-6 & U: GTCTATACCACTTCACAAGTCGGA & 441 \\
& D: TTGGATGGTCTTGGTCCTTAGCCA & \\
$\alpha$-actin & U: GAAATCGTGGGTGACATCAAA & 478 \\
& D: ACTCATCGTACTCCTGCTTGCTGA & \\
\hline
\end{tabular}

PCR, polymerase chain reaction; TNF- $\alpha$, tumor necrosis factor- $\alpha$; IL-6, interleukin-6; U, upstream; D, downstream.

USA) for isolation of total RNA from vessels. The samples were treated with a DNase I kit as follows: $2 \mu \mathrm{g}$ total RNA, $2 \mu 1$ buffer $(10 \mathrm{X})$, and $2 \mu \mathrm{l}$ DNase I were incubated for $10 \mathrm{~min}$ at $65^{\circ} \mathrm{C}$. The conditions for cDNA synthesis were as follows: $65^{\circ} \mathrm{C}$ for $5 \mathrm{~min}$ and $4^{\circ} \mathrm{C}$ for $2 \mathrm{~min}$. The PCR reaction contained $4 \mu \mathrm{l}$ of $5 \mathrm{X}$ RT Buffer (Takara, Bio, Inc., Otsu, Japan), $2 \mu \mathrm{l}$ of $0.1 \mathrm{M}$ DTT, $1 \mu \mathrm{l}$ of $10 \mathrm{mM}$ dNTPs, $1 \mu \mathrm{l}$ of HiFi-MMLV enzyme mix, and $2 \mu \mathrm{l}$ of primers. ROX plus and SYBR Premix Ex Taq II were used for RT-PCR. The thermal profile was as follows: $95^{\circ} \mathrm{C}$ for $5 \mathrm{sec}$ (30 cycles), and $60^{\circ} \mathrm{C}$ for $40 \mathrm{sec}(45$ cycles). The primer sequences are shown in Table I.

Statistical analysis. Data were analyzed using SPSS version 17.0 software (SPSS, Inc., Chicago, Il, USA). All data are presented as mean \pm standard deviation. Comparisons between groups were by independent t-test. Comparisons among groups were by analysis of variance (ANOVA) followed by an LSD test. $\mathrm{P}<0.05$ was considered to indicate a statistically significant difference.

\section{Results}

Determination of optimal parameters for electrocautery. The rabbits were euthanized in preliminary experiments and the abdominal aortas after treatment were examined by immunofluorescence staining of frozen sections. Combined with visual comparison, immunofluorescence staining showed that with the $1 \mathrm{~W}+1 \mathrm{~S}$ power and activation time setting, vascular morphology was not affected. In addition, samples treated with the $1 \mathrm{~W}+1 \mathrm{~S}$ setting had smooth vascular lumens, which was the optimal effect of electrocautery in the rabbit model (Fig. 3). Increasing the power and activation time settings lead to more impaired results. In some vessels, electrocautery caused obvious thermal damage to the vascular structure, according to the comparisons by visual examination and immunofluorescence (Fig. 4). 


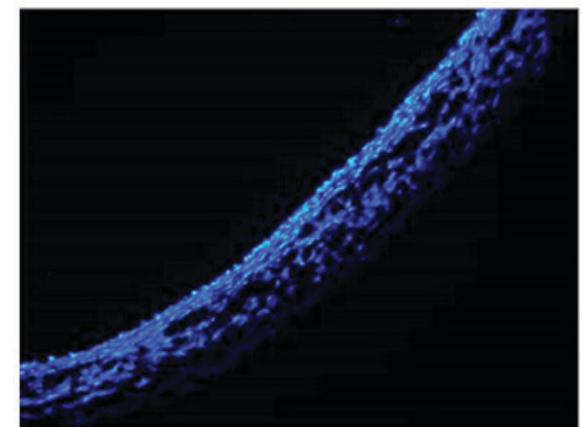

$1 \mathrm{~W}+1 \mathrm{~S}$

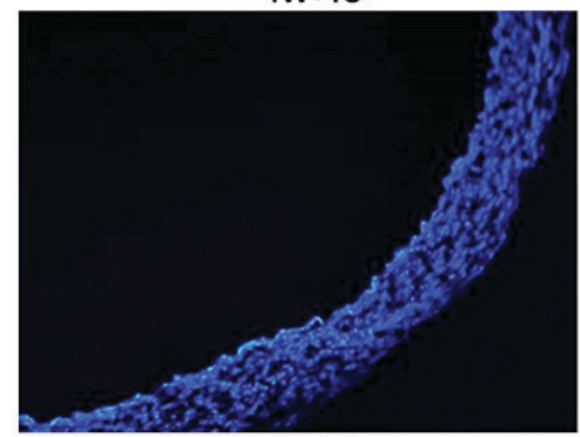

$2 \mathrm{~W}+1 \mathrm{~S}$

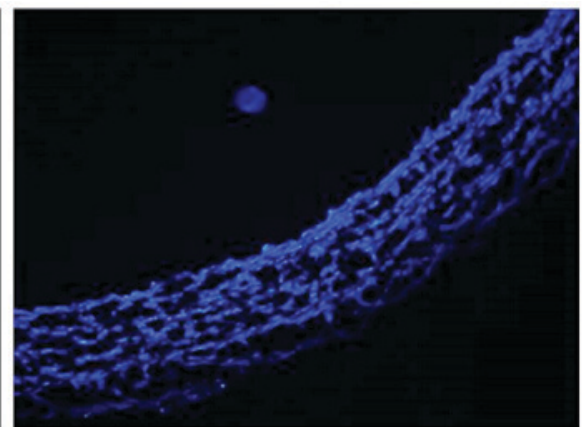

$1 \mathrm{~W}+2 \mathrm{~S}$

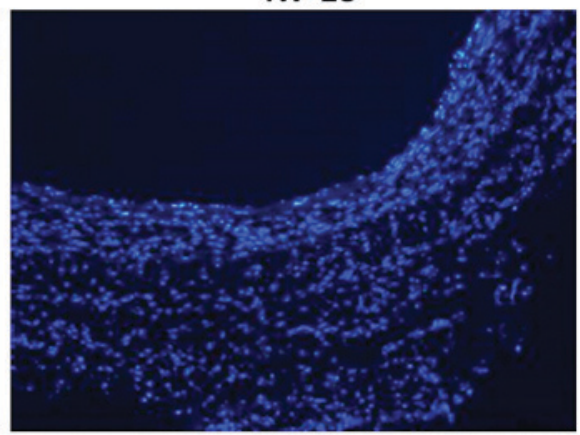

$2 \mathrm{~W}+2 \mathrm{~S}$

Figure 3. Electrocautery plan option (immunofluorescence compassion).

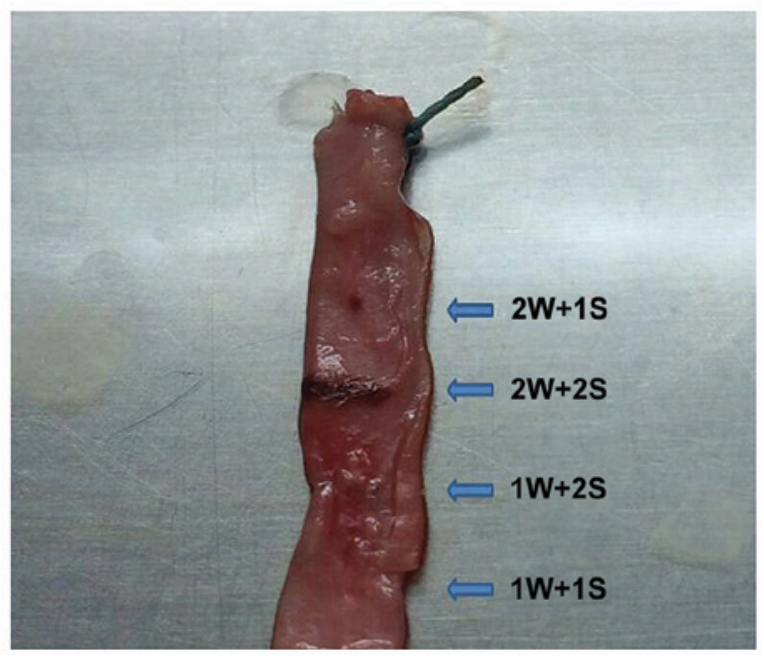

Figure 4. Electrocautery plan option (visual compassion).

Endarterectomy + electrocautery reduces intimal injury of the abdominal aorta. Aortic tissues were examined histologically to clarify the mechanism of the effect of electrocautery on blood flow after surgery. Immunofluorescence showed that the lumens of abdominal aortas in endarterectomy + electrocautery group were significantly smoother than those of the control group (Fig. 5A-E). These findings demonstrated that endarterectomy + electrocautery reduces intimal injury of the abdominal aorta by making the vascular lumen smooth without protuberance.

Endarterectomy + electrocautery improves postoperative blood flow. All rabbits survived and recovered well after surgery. None required ventilation because of airway occlusion. There was no significant difference in blood flow velocity after surgery between the sham and study groups $(808 \pm 39.4$ vs. $793 \pm 27.8 \mathrm{~mm} / \mathrm{sec}, \mathrm{P}>0.05)$, whereas the velocity of blood flow in the control group was significantly lower than in the study group $(728 \pm 31.5$ vs. $793 \pm 27.8 \mathrm{~mm} / \mathrm{sec}, \mathrm{P}<0.05)$, suggesting successful establishment of the rabbit model of endarterectomy + electrocautery. Interestingly, 20 days after surgery, the velocity of blood flow in the control group decreased significantly. However, blood flow in the study group was significantly higher than in the control group $(660 \pm 31.7$ vs. $758 \pm 23.9 \mathrm{~mm} / \mathrm{sec}, \mathrm{P}<0.05)$. Furthermore, vessel diameter was significantly lower in the control group compared with the study group 20 days after surgery $(3.4 \pm 0.4$ vs. $4.0 \pm 0.7 \mathrm{~mm}, \mathrm{P}<0.05)$. Taken together, these results indicate that endarterectomy+electrocautery improves postoperative blood flow.

Endarterectomy + electrocautery reduces the rate of apoptosis of vascular ECs. Flow cytometric analysis of ECs demonstrated that both the study and control groups had higher rates of apoptosis than the sham group ( $\mathrm{P}<0.05$, Fig. 6$)$, while the range of the rise in the study group was significantly lower than that in the control group over time ( $\mathrm{P}<0.05$, Fig. 6$)$. These results further suggested that endarterectomy + electrocautery attenuates apoptosis of ECs postoperatively. Therefore, the electrocautery-mediated effects on blood flow after surgery appears to be associated with decreased intimal injury of the abdominal aorta and decreased rate of apoptosis of vascular ECs.

Endarterectomy + electrocautery reduces $T N F-\alpha$ and IL-6 expression and inflammation. To further explore the mechanism related to the favorable effects of electrocautery, the vascular levels of TNF- $\alpha$ and IL- 6 were measured by ELISA 
A

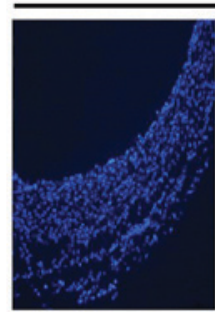

Sham

C

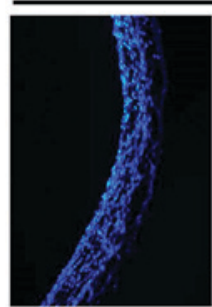

Sham

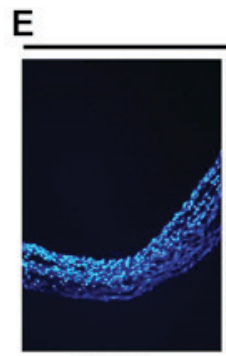

Sham
Day 0

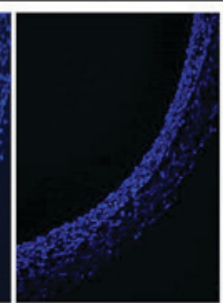

Control

Day 3

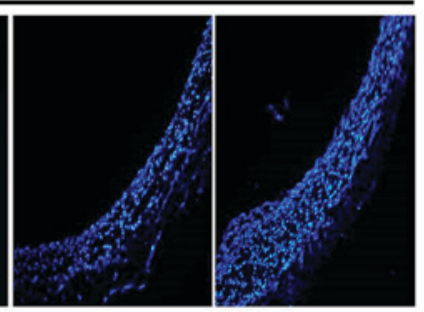

Control

Day 20

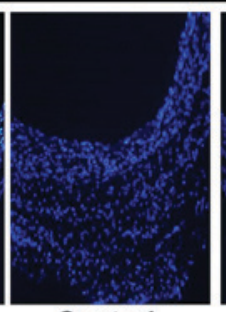

Control

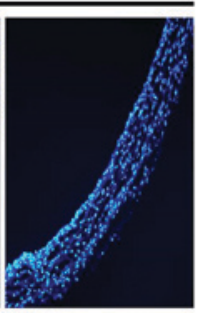

Study

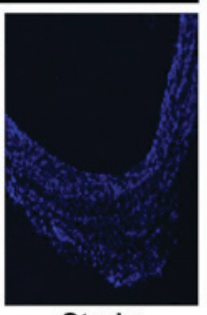

Study

B

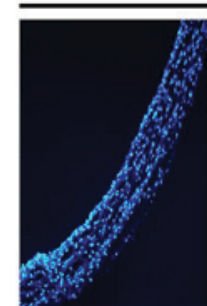

Sham

D

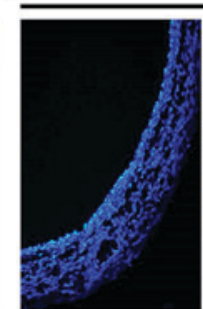

Sham

Day 1

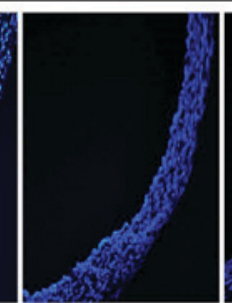

Control

Day 7

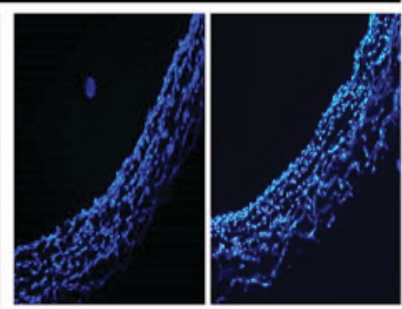

Control

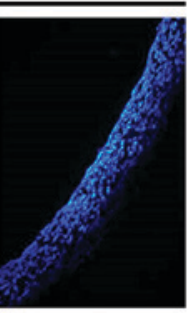

Study

Study

Figure 5. Immunofluorescence staining of three groups at (A) day 0,(B) day 1, (C) day 3, (D) day 7 and (E) day 20.

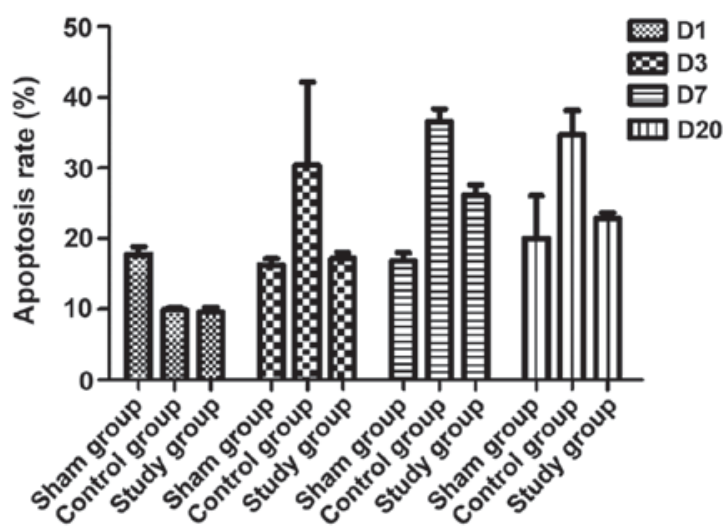

Figure 6. Apoptosis rate of vascular ECs. ECs, endothelial cells.

and qRT-PCR. Our ELISA results showed that the levels of TNF- $\alpha$ and IL- 6 in serum were significantly increased in the control group, while they decreased gradually compared with the sham group. In the study group, the postoperative levels of TNF- $\alpha$ and IL- 6 were similar to normal serum levels, and they remained unchanged over time compared with the sham group $(\mathrm{P}<0.05$, Fig. 7$)$. Excessive inflammation was shown to be related to intimal hyperplasia. Electrocautery significantly reduced TNF- $\alpha$ and IL- 6 expression in vessel tissue in the control group, and their expression decreased gradually compared with the sham group. Their expression was maintained at almost normal levels postoperatively, and there
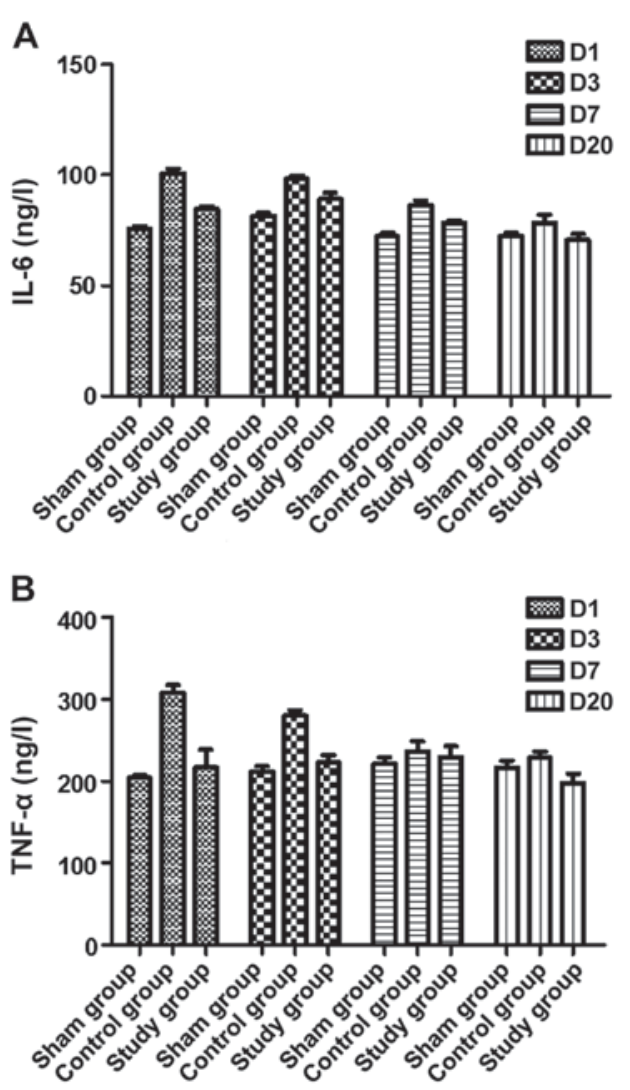

Figure 7. (A) TNF- $\alpha$ and (B) IL-6 protein expression in different groups. TNF- $\alpha$, tumor necrosis factor- $\alpha$; IL-6, interleukin- 6 . 
A

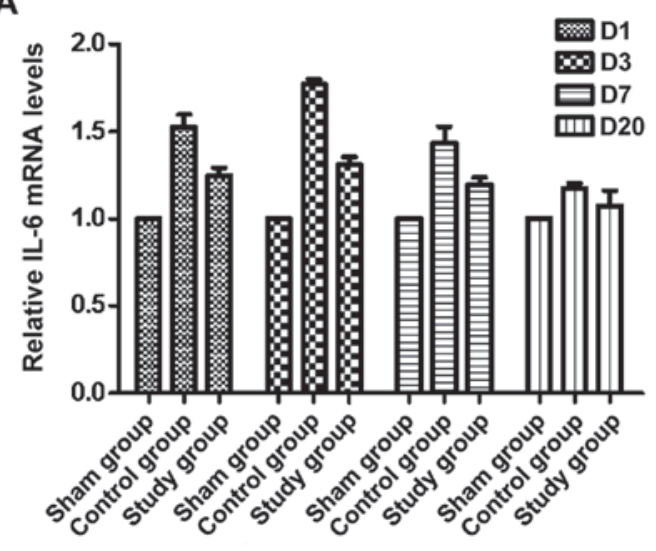

B

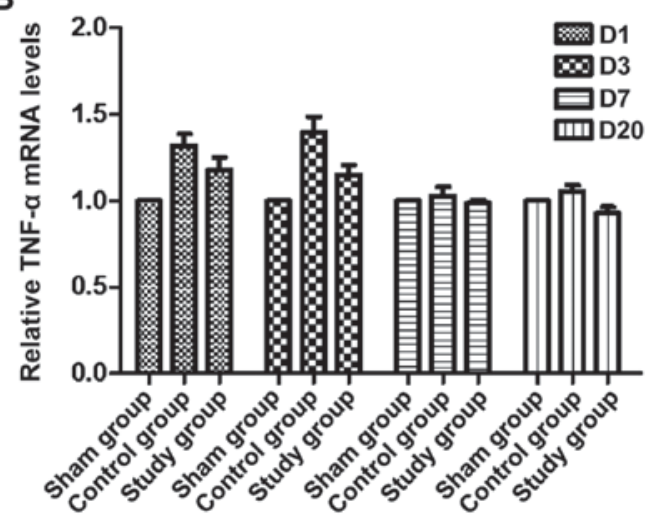

Figure 8. TNF- $\alpha$ (A) and IL-6 (B) mRNA expression in different group. TNF- $\alpha$, tumor necrosis factor- $\alpha$; IL-6, interleukin- 6 .

were no significant differences over time ( $\mathrm{P}<0.05$, Fig. 8). These results indicate that electrocautery alleviated inflammation in rabbits.

\section{Discussion}

CE is widely applied to treat diffuse coronary artery disease (7). Previous evidence suggested that the long-term value of CE combined with off-pump CABG was promising (8). However, recent studies arrived at different conclusions. A study suggested that the rate of mortality of patients who underwent $\mathrm{CABG}$ combined with $\mathrm{CE}$ was significantly higher than those who were treated by CABG or CE alone (9). The fact that patients who require $\mathrm{CE}$ treatment are commonly at a higher risk of perioperative MI and death may contribute to the higher rates of morbidity and mortality associated with CE (2).

CE-related increases in inflammation and thrombosis may be associated with embolization and thrombogenesis from dislodged atheromatous material (2). CE commonly injures vascular endothelium and causes mechanical injury to the coronary vessels. Endothelial dysfunction may result in increased thrombogenesis and atheroembolization, and reduction in graft flow, which consequently leads to thrombosis and postoperative inflammation. The release of inflammatory factors (such as IL-6 and TNF- $\alpha$ ) was enhanced following the extent of endothelial damage. To accommodate shear stress and high wall stress, arteries without an intima undergo postoperative remodeling. The remodeling is characterized by endothelial proliferation and is affected by platelet aggregation. The process of thrombogenesis may contribute to vascular occlusion or stenosis, leading to bypass graft failure (10). Furthermore, endothelial proliferation may balance the remodeling process by covering the exposed coarse vessel wall induced by mechanical injury from endarterectomy.

Immediate responses occur after acute traumatic damage to control injury and trigger the recovery process, including the release of inflammatory factors. TNF- $\alpha$ is an important inflammatory mediator that can activate cell signaling and induce the release of IL-6 (11). The activation and aggregation of neutrophils are increased by IL- 6 , and are used as sensitive indexes of vascular endothelial injury and inflammation (12). Our study showed that serum TNF- $\alpha$ and IL-6 in the control group reached peak levels on approximately postoperative day 7 , and decreased to normal levels on day 20 . In the study group, the levels increased slightly and decreased to normal on day 7. The IL- 6 and TNF- $\alpha$ gene expression showed similar trends. Therefore, monitoring the variation in the levels of IL- 6 and TNF- $\alpha$ can help estimate the degree of postoperative injury in animal models or clinical trials.

Electrocautery with electrocoagulation electrotomes is emerging for use in various clinical procedures, as a self-modified strategy to prevent excessive inflammation and thrombosis (13). However, technical challenges associated with the power and activation time of electrocautery limit the applications in animals and in the clinic (14). Although the application of endarterectomy on arteries appears to exert protective effects via endothelial coverage and smoothing of coarse vessel lumens, high power and activation time settings may cause severe thermal and excessive damage in animal models (13). The aim of the preliminary experiments was to limit the range of electrocautery within the adventitia. Only flattening effects occurred.

During the early phase of post-endarterectomy adaptation, the media and adventitia are exposed to high wall shear stress and the intense pulsatile stretch force from the arterial circulation. Marked increases in mechanical stretch on coarse vascular lumens resulting from exposure to the arterial circulation has been demonstrated to induce apoptosis of ECs in a sheep model of open carotid endarterectomy (5). This mechanical stretch-induced vascular injury may contribute to the subsequent excessive inflammatory response and rapid EC apoptosis, which can ultimately result in stenosis and thrombosis (6). Brown et al (15) found that vessel wall injury induced changes in blood flow dynamics in a rat model of carotid endarterectomy. The electrocautery likely caused a tissue flattening effect in the abdominal aorta after endarterectomy to prevent mechanism-induced shear stress injury. However, the duration of the electrocautery use in rabbits is unknown. Electrocautery can be considered a double-edged sword in that it causes both severe thermal injury and vascular lumen improvement after abdominal aortic endarterectomy. Therefore, we established the protocol in rabbits with appropriate power and activation time. Our study showed that intravascular electrocautery in the abdominal aorta significantly improved blood flow 20 days after surgery compared with the isolated endarterectomy group. The beneficial effects of endarterectomy + electrocautery on blood flow appeared to be associated with smooth 
vascular lumens and reduced inflammation. Compared with isolated application of endarterectomy, endarterectomy + electrocautery has substantial advantages. It can not only be managed without side effects such as thermal injury, but can also ameliorate inflammation after surgery, thus, protecting vessels from vascular restenosis (6). In this study, we found that intravascular application of electrocautery significantly reduced the serum expression of TNF- $\alpha$ and vascular expression of IL-6. In addition, electrocautery was quite safe. In this study, obvious electrocautery-related injury responses in rabbits were not observed, although there are several reports of side effects from electrocautery in different clinical studies and animal models $(13,16)$.

In our study, to evaluate the effects of endarterectomy + electrocautery on the abdominal aorta in a rabbit model, we explored the levels of pro-inflammatory factors, measured arterial flow parameters, and examined graft histology 20 days after surgery. More et al (17) evaluated changes in the vessel wall in a rabbit model of balloon angioplasty and showed that thickened endothelium was observed at day 7 and peaked at 4 weeks. Therefore, 20 days after surgery appears to be the ideal time point for assessing the effects of electrocautery on arterial flow and histology. Given that our results showed that electrocautery reduced inflammation and improved vessel flow 20 days after surgery in the model of abdominal aortic endarterectomy, we believe that the clinical use of endarterectomy + electrocautery in CABG is a promising approach. In the rabbit model, electrocautery can only partially alleviate postoperative intravascular resistance. The long-term effects of electrocautery on arterial remodeling remain unknown. Additionally, a pig model of artery bypass grafting appears to be more appropriate for the observation of long-term effects because of the high similarity in coronary circulation between humans and pigs. Currently, we are using a pig model to explore the effects of electrocautery on remodeling of the coronary artery. However, the activation time and precise power of electrocautery in animals varies depending on the size, weight, and species.

In conclusion, we found that intravascular application of electrocautery reduced thrombosis and inflammation 4 weeks post-artery bypass grafting in a rabbit model. The favorable effects appeared to be associated with smoothed vascular lumens, decreased inflammation, and decreased EC apoptosis. Although the short-term effects of intravascular application of electrocautery appear to be promising, the long-term effects of electrocautery on arterial remodeling and the clinical value of electrocautery in $\mathrm{CE}$ require further exploration.

\section{Acknowledgements}

This study was supported by the project of Basic and Clinical Research Cooperation From Capital Medical University (no. 16JL05) and the Yangfan Project of Clinical Technical Innovation From the Hospital Authority of Beijing (no. XM201312).

\section{References}

1. Sirivella S, Gielchinsky I and Parsonnet V: Results of coronary artery endarterectomy and coronary artery bypass grafting for diffuse coronary artery disease. Ann Thorac Surg 80: 1738-1744, 2005.

2. Soylu E, Harling L, Ashrafian H, Casula R, Kokotsakis J and Athanasiou T: Adjunct coronary endarterectomy increases myocardial infarction and early mortality after coronary artery bypass grafting: A meta-analysis. Interact Cardiovasc Thorac Surg 19: 462-473, 2014.

3. Soylu E, Harling L, Ashrafian H, Casula R, Kokotsakis J and Athanasiou T: Adjunct coronary endarterectomy increases myocardial infarction and early mortality after coronary artery bypass grafting: a meta-analysis. Interact Cardiovasc Thorac Surg 19: 462-473, 2014.

4. Atik FA, Dallan LA, de Oliveira SA, Lisboa LA, Platania F, Cabral RH and Jatene AD: Myocardial revascularization with coronary endarterectomy. Stratification of risk factors for early mortality. Arq Bras Cardiol 75: 269-280, 2000.

5. Bezon E, Khalifa AA, Le Gal G, Choplain JN, Mansourati J and Barra JA: Use of arterial patch to improve re-endothelialization in a sheep model of open carotid endarterectomy. An incentive to use internal thoracic artery as an on-lay patch following coronary endarterecomy? Interact Cardiovasc Thorac Surg 8: 543-547, 2009.

6. Liang JJ, Xue W, Lou LZ, Liu C, Wang ZF, Li QG and Huang SH: Correlation of restenosis after rabbit carotid endarterectomy and inflammatory cytokines. Asian Pac J Trop Med 7: 231-236, 2014.

7. Bailey CP, May A and Lemmon WM: Survival after coronary endarterectomy in man. J Am Med Assoc 164: 641-646, 1957.

8. Vohra HA, Kanwar R, Khan T and Dimitri WR: Early and late outcome after off-pump coronary artery bypass graft surgery with coronary endarterectomy: A single-center 10-year experience. Ann Thorac Surg 81: 1691-1696, 2006.

9. Tiruvoipati R, Loubani M, Lencioni M, Ghosh S, Jones PW and Patel RL: Coronary endarterectomy: Impact on morbidity and mortality when combined with coronary artery bypass surgery. Ann Thorac Surg 79: 1999-2003, 2005.

10. Liu SQ, Ruan YY, Tang D, Li YC, Goldman J and Zhong L: A possible role of initial cell death due to mechanical stretch in the regulation of subsequent cell proliferation in experimental vein grafts. Biomech Model Mechanobiol 1: 17-27, 2002.

11. Wainwright CL, Miller AM and Wadsworth RM: Inflammation as a key event in the development of neointima following vascular balloon injury. Clin Exp Pharmacol Physiol 28: 891-895, 2001.

12. Holub Z, Jabor A, Sprongl L, Kliment L, Fischlová D and Urbánek S: Inflammatory response and tissue trauma in laparoscopic hysterectomy: Comparison of electrosurgery and harmonic scalpel. Clin Exp Obstet Gynecol 29: 105-109, 2002.

13. Fiorelli A, Accardo M, Carelli E, Del Prete A, Messina G, Reginelli A, Berritto D, Papale F, Armenia E, Chiodini P, et al: Harmonic technology versus neodymium-doped yttrium aluminium garnet laser and electrocautery for lung metastasectomy: An experimental study. Interact Cardiovasc Thorac Surg 23: 47-56, 2016.

14. Robinson AM, Fishman AJ, Bendok BR and Richter CP: Functional and physical outcomes following use of a flexible $\mathrm{CO}_{2}$ laser fiber and bipolar electrocautery in close proximity to the rat sciatic nerve with correlation to an in vitro thermal profile model. BioMed Res Int 2015: 280254, 2015.

15. Brown AT, Chen H, Davis JA, Qureshi I, Cruz CP, Poirier LA, Eidt JF and Moursi MM: Plasma homocysteine measurements after carotid artery manipulation and clamping in a rat CEA model. J Vasc Surg 40: 796-802, 2004.

16. Uluyol S, Karakaya NE, Gur MH, Kilicaslan S, Kantarcioglu EO, Yagiz O and Arslan IB: Radiofrequency thermal ablation versus bipolar electrocautery for the treatment of inferior turbinate hypertrophy: Comparison of efficacy and postoperative morbidity. Int Arch Otorhinolaryngol 20: 2-5, 2016.

17. More RS, Rutty G, Underwood MJ, Brack MJ and Gershlick AH: A time sequence of vessel wall changes in an experimental model of angioplasty. J Pathol 172: 287-292, 1994. 